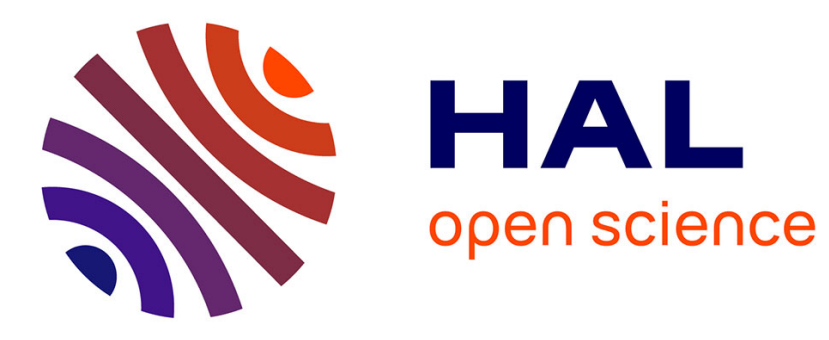

\title{
Does JavaScript software embrace classes?
}

Leonardo Humberto Silva, Miguel Ramos, Marco Tulio Valente, Alexandre Bergel, Nicolas Anquetil

\section{To cite this version:}

Leonardo Humberto Silva, Miguel Ramos, Marco Tulio Valente, Alexandre Bergel, Nicolas Anquetil. Does JavaScript software embrace classes?. SANER 2015: International Conference on Software Analysis, Evolution, and Reengineering, Mar 2015, Montreal, Canada. pp.73 - 82, 10.1109/SANER.2015.7081817 . hal-01185854

\section{HAL Id: hal-01185854 \\ https://inria.hal.science/hal-01185854}

Submitted on 21 Aug 2015

HAL is a multi-disciplinary open access archive for the deposit and dissemination of scientific research documents, whether they are published or not. The documents may come from teaching and research institutions in France or abroad, or from public or private research centers.
L'archive ouverte pluridisciplinaire HAL, est destinée au dépôt et à la diffusion de documents scientifiques de niveau recherche, publiés ou non, émanant des établissements d'enseignement et de recherche français ou étrangers, des laboratoires publics ou privés. 


\section{Does JavaScript Software Embrace Classes?}

\author{
Leonardo Humberto Silva, Miguel Ramos, \\ Marco Tulio Valente \\ Federal University of Minas Gerais, Brazil \\ \{leonardosilva,miguel.ramos,mtov\}@dcc.ufmg.br
}

\author{
Alexandre Bergel \\ Pleiad Lab \\ University of Chile \\ abergel@dcc.uchile.cl
}

\author{
Nicolas Anquetil \\ RMoD Project-Team \\ INRIA Lille Nord Europe, France \\ nicolas.anquetil@inria.fr
}

\begin{abstract}
JavaScript is the de facto programming language for the Web. It is used to implement mail clients, office applications, or IDEs, that can weight hundreds of thousands of lines of code. The language itself is prototype based, but to master the complexity of their application, practitioners commonly rely on some informal class abstractions. This practice has never been the target of empirical investigations in JavaScript. Yet, understanding it would be key to adequately tune programming environments and structure libraries such as they are accessible to programmers. In this paper we report a large and indepth study to understand how class emulation is employed in JavaScript applications. We propose a strategy to statically detect class-based abstractions in the source code of JavaScript systems. We used this strategy in a dataset of 50 popular JavaScript applications available from GitHub. We found systems structured around hundreds of classes, suggesting that JavaScript developers are standing on traditional class-based abstractions to tackle the growing complexity of their systems.
\end{abstract}

\section{INTRODUCTION}

JavaScript is the de facto programming language for the Web [1]. The language was initially designed in the mid1990s to extend web pages with small executable code. Since then, its popularity and relevance has only grown [2], [3]. For example, JavaScript is now the most popular language at GitHub, considering new repositories created by language. It is also reported that the language is used by 97 out of the web's 100 most popular sites [4]. Concomitantly with its increasing popularity, the size and complexity of JavaScript software is in steady growth. The language is now used to implement mail clients, office applications, IDEs, etc, which can reach several hundred thousands lines of code ${ }^{1}$.

Despite the complexity, size, and relevance of modern JavaScript software, little research investigated how developers effectively organize and manage large JavaScript software systems. Specifically, JavaScript is an imperative, and objectoriented language centered on prototypes, rather than being a class-based language [1], [5], [6]. Despite not having explicit class constructions, the prototype-based object system of the language is flexible enough to support the implementation of mainstream class-based abstractions, including attributes, methods, constructors, inheritance hierarchies, etc. However, structuring a software around such abstractions is a design decision, which should be taken by JavaScript developers. In other words, the language is flexible enough to support different

\footnotetext{
${ }^{1}$ http://sohommajumder.wordpress.com/2013/06/05/gmail-has-biggestcollection-of-javascript-code-lines-in-the-world, verified 11/15/2014
}

modularization paradigms, including procedural programming (e.g., a system is as set of functions, like in C), modular programming (e.g., a system is a set of modules that encapsulate data and operations, like in Modula-2), and class-based objectoriented programming (e.g., a system is a set of classes, like in Java).

In this paper, we report an empirical study conducted to shed light on how often JavaScript developers modularize their systems around abstractions that resemble object-oriented classes. An in-depth understanding of this phenomena can contribute to the design of better programming environments for JavaScript, with support for example to class-based program views. It can also contribute to specifying tools for helping JavaScript developers on designing, understanding, and evolving JavaScript classes. Finally, the new standard version of JavaScript, named ECMAScript 6, will include syntactical support for classes [7]. Therefore, revealing how JavaScript developers currently emulate classes might be a valuable information for developers that plan to use classes in their systems, according to this new standard syntax.

The main contributions of our work are as follows:

- We document how prototypes in JavaScript are used to support the implementation of structures including both data and code and that are further used as a template for the creation of objects (Section II). In this paper, we use the term classes to refer to such structures, since they have a very similar purpose than the native classes available in mainstream object-oriented languages.

- We describe a strategy to statically identify classes in the code of JavaScript software, as described in Section III.

- We propose an open-source supporting tool, called JSCLASSFINDER, that practitioners can use to detect and inspect classes in JavaScript software. This tool is described in Section III-B

- We provide a thorough study on the usage of classes in a dataset of 50 popular JavaScript software available at GitHub (Section IV). This study answers the following research questions: (a) How often JavaScript developers use classes? (b) How often JavaScript developers use inheritance? (c) What is the size of JavaScript classes (in terms of number of methods and attributes)?

\section{Classes in JaVaScript}

This section lists the different mechanisms to emulate classes in JavaScript. To describe these mechanisms we conducted an 
informal survey on documents available in the web, including tutorials ${ }^{2}$, blogs ${ }^{3}$, and StackOverflow discussions ${ }^{4}$. We also surveyed a catalogue of five encapsulation styles for JavaScript proposed by Gama et al. [8] and JavaScript books targeting language practitioners [9], [10].

Basically, objects in JavaScript are sets of name-value pairs. The names are strings, called properties, and the values are numbers, booleans, strings, functions, etc. To implement classes in JavaScript - i.e., data structures that resemble the class concept of mainstream object-oriented languages - the most common strategy is to use functions. Particularly, any function can be used as template for the creation of objects. In a function, the keyword this is used to define properties that emulate attributes and methods. Attributes are properties associated to numbers, booleans, strings, etc. Methods are properties associated to inner functions. The keyword new is used to create objects for a class.

To illustrate the definition of classes in JavaScript, we use a simple Circle class. Listing 1 presents the function that defines this class (lines 1-6), which includes a radius attribute and a getArea method.

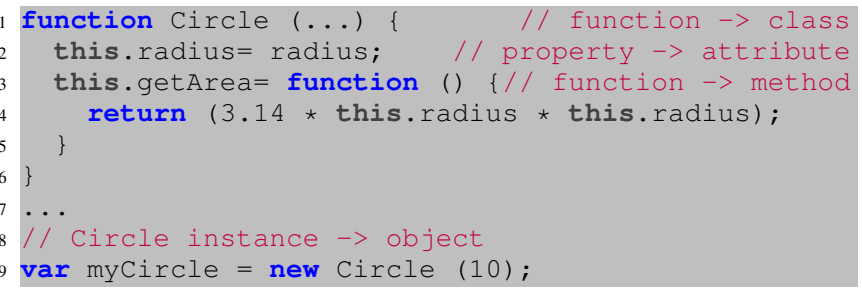

Listing 1. Class declaration and object instantiation

Each object in JavaScript has a default prototype property that refers to another object. To evaluate an expression like obj.p, the runtime starts searching for property $\mathrm{p}$ in obj, then in obj.prototype, then in obj.prototype.prototype, and so on until it finds the desired property or reaches an empty object. When an object is created using new $\mathrm{C}$ its prototype is set to the prototype of the function $\mathrm{C}$, which by default is defined as pointing to an Object. Therefore, a chain of prototype links usually ends at Object.

By manipulating the prototype property, we can define a method whose implementation is shared by all object instances. It is also possible to define properties shared by all objects of a given class, akin to static attributes in classbased languages. In listing 2, Circle includes a pi static attribute and a getCircumference method. It is worth noting that getCircumference is not a method attached to the class (as a static method in Java). It has for example access to the this variable, whose value is not determined using lexical scoping rules, but instead using the caller object.

\footnotetext{
${ }^{2} \mathrm{https}: / /$ developer.mozilla.org/en-US/docs/Web/JavaScript/Introduction_to_ Object-Oriented_JavaScript

${ }^{3} \mathrm{http}: / /$ javascript.crockford.com/prototypal.html

${ }^{4} \mathrm{http} / / /$ stackoverflow.com/questions/387707/whats-the-best-way-to-definea-class-in-javascript
}

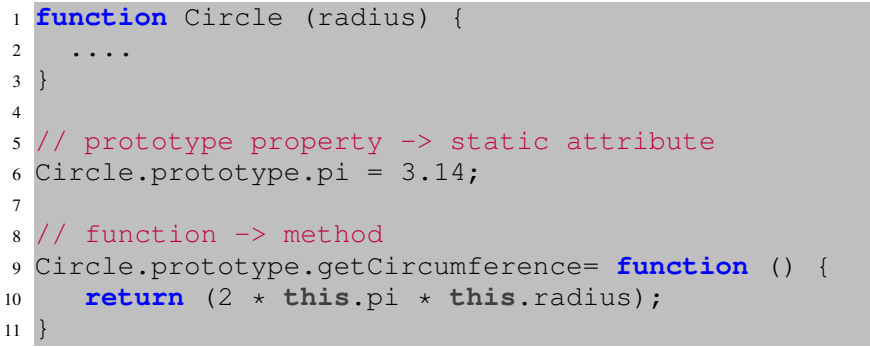

Listing 2. Using prototype to define methods and static attributes

Prototypes are also used to simulate inheritance hierarchies. In JavaScript, we can consider that a class $\mathrm{C} 2$ is a subclass of C1 if C2's prototype refers to an instance of C1. For example, Listing 3 shows a class Circle2D that extends Circle with its position in a Cartesian plane.

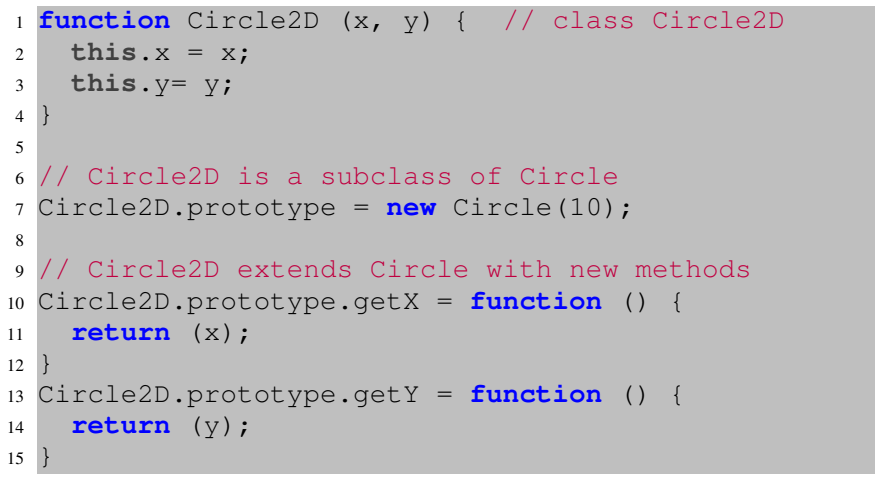

Listing 3. Implementing subclasses

Alternatively, the subclass may refer directly to the prototype of the superclass, which is possible using the Object.create() method. This method creates a new object with the specified prototype object, as illustrated by the following code:

\section{Circle2D.prototype=0bject. create (Circle.prototype)}

Table I summarizes the strategy presented in this section to map class-based object-oriented abstractions to JavaScript abstractions.

TABLE I

CLASS-BASED LANGUAGES VS JAVASCRIPT

\begin{tabular}{ll}
\hline Class-based languages & JavaScript \\
\hline Class & Function \\
Attribute & Property \\
Method & Inner function \\
Static attribute & Prototype property \\
Inheritance & Prototype chaining \\
\hline
\end{tabular}

\section{Detecting Classes In JaVaScRipt}

In this section, we describe a strategy to statically detect classes in JavaScript source code (Section III-A). Section III-B describes the tool we implemented to detect classes in 
JavaScript using the proposed strategy. We also report limitations of this strategy, mainly due to the dynamic behavior of JavaScript (Section III-C).

\section{A. Strategy to Detect Classes}

To detect classes, we reused with minimal adaptations a simple grammar for JavaScript, originally proposed by Anderson $e t$ al. [11] to represent the way objects are created in JavaScript and the way objects acquire fields and methods. This grammar is as follows:

$$
\begin{aligned}
\text { Program }::= & \text { FuncDecl } \\
\text { FunDecl }::= & \text { function } \mathrm{f}()\{\operatorname{Exp}\} \\
\text { Exp }::= & \text { new } \mathrm{f}() \\
& \text { this.a=Exp } \\
& \text { this.a= function }\{\operatorname{Exp}\} \\
& \text { f.prototype.a=Ex } ; \\
& \text { f.prototype.a= function }\{\operatorname{Exp}\} \\
& \text { f.prototype new }() ; \\
& \text { Object.create(f.prototype); }
\end{aligned}
$$

This grammar assumes that a program is composed of functions, and that a function's body is an expression. The expressions of interest are the ones to create objects and to add properties to functions via this or prototype.

Definition \#1: A class is a tuple $(C, \mathcal{A}, \mathcal{M})$, where $C$ is the class name, $\mathcal{A}=\left\{a_{1}, a_{2}, \ldots, a_{p}\right\}$ are the attributes defined by the class, and $\mathcal{M}=\left\{m_{1}, m_{2}, \ldots, m_{q}\right\}$ are the methods. Moreover, a class $(C, \mathcal{A}, \mathcal{M})$, defined in a program $P$, must respect the following conditions:

- $P$ must have a function with name $\mathrm{C}$.

- $P$ must include at least one expression new $\mathrm{C}()$.

- For each $a \in \mathcal{A}$, the function $\mathrm{C}$ must include an assignment this.a $=\operatorname{Exp}$ or $P$ must include an assignment C.prototype.a $=$ Exp.

- For each $m \in \mathcal{M}$, the function $\mathrm{C}$ must include an assignment this.m $=$ function $\{\operatorname{Exp}\}$ or $P$ must include an assignment C.prototype.m $=$ function $\{\operatorname{Exp}\}$.

In the JavaScript examples of Section II we have two classes:

- (Circle,\{radius,pi $\},\{$ getArea,getCircumference $\})$

- (Circle2D, $\{\mathrm{x}, \mathrm{y}\}$, \{getX, getY $\})$

Definition \#2: Assuming that $(C 1, \mathcal{A} 1, \mathcal{M} 1)$ and $(C 2, \mathcal{A} 2, \mathcal{M} 2)$ are classes in a program $P$, we define that $C 2$ is a subclass of $C 1$ if one of the following conditions holds:

- $P$ includes an assignment $\mathrm{C} 2$.prototype = new $\mathrm{C} 1()$.

- $P$ includes an assignment C2.prototype = Object.create(C1.prototype).

In the examples of Section II, Circle2D is a subclass of Circle.

\section{B. Tool Support}

We implemented a tool, called JSCLASSFINDER, for identifying classes in JavaScript programs. As illustrated in Figure 1, this tool works in two steps. In the first step, Esprima ${ }^{5}-\mathrm{a}$ widely used JavaScript Parser-is used to generate a full abstract syntax tree, in JSON format. In the second step, we implemented an application that supports the strategies described in Section III-A to detect classes in a JavaScript AST in the JSON format. JSCLASSFINDER also collects the following basic object oriented metrics: Number of Attributes (NOA), Number of Methods (NOM), Depth of Inheritance Tree (DIT), and Number of Children (NOC) [12].

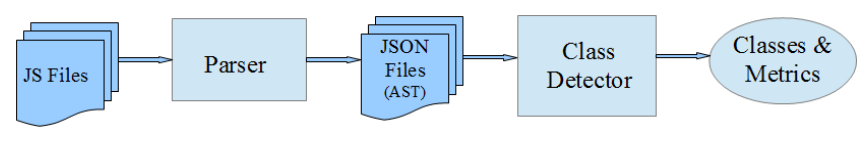

Fig. 1. JSClassFinder

\section{Limitations}

The proposed strategy requires each class $C$ to have at least one corresponding new $\mathrm{C}$ expression in the program. This is important because any function in JavaScript has access to this and can use it to add properties to the calling object or to the global context. For example, suppose the following code:

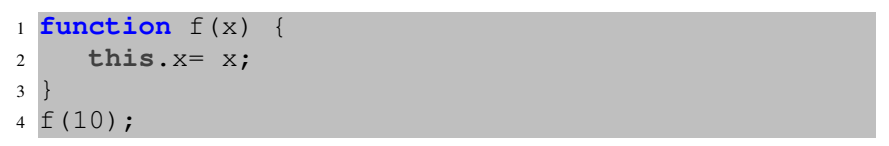

The call to $f$ does not have a target object. In this case, the result is to add a property $\mathrm{x}$ in the object that represents the global context in JavaScript programs. Therefore, although $f$ resembles a constructor function, it is not in fact used as a template to create objects, since the program does not include a new. For this reason, it is not classified as a class, according to our definition. On the other hand, classes designed to be instantiated by client applications, as would be the case of the public interface of APIs, are not detected by the proposed strategy. In this case, the call to new is typically made by the clients.

We also acknowledge that there is not one single strategy to emulate classes in JavaScript. For example, it is possible to create "singleton" objects directly, without using any class-like construction, as in this example:

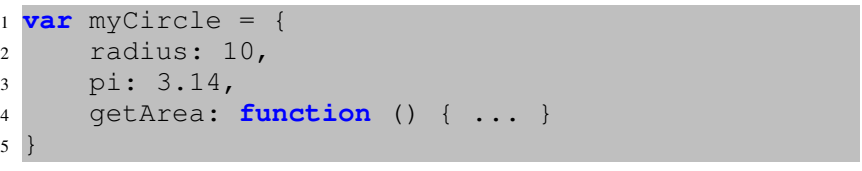

In addition, there are numerous JavaScript frameworks, like Prototype $^{6}$ and AngularJS ${ }^{7}$, that support their own style for implementing class-like abstractions. For this reason, we do

\footnotetext{
${ }^{5}$ http://esprima.org/

${ }^{6} \mathrm{http}: / /$ prototypejs.org

${ }^{7}$ https://angularjs.org
} 
not struggle to cover the whole spectrum of alternatives to implement classes. Instead, we consider only the strategy closest to the syntax and semantics of class-based languages. Recognizing other ways to mimic classes could be the goal of some future work.

Moreover, there are abstractions related to classes that are more difficult to emulate in JavaScript, like abstract classes and interfaces. Encapsulation is another concept that does not have a straightforward mapping to JavaScript. A common workaround to simulate private members in JavaScript is by using local variables and closures. As shown in Listing 4 , an inner function $\mathrm{f} 2 \mathrm{in} \mathrm{JavaScript}$ has access to the variables of its outer function $\mathrm{f} 1$, even after $\mathrm{f} 1$ returns. Therefore, local variables declared in $\mathrm{f} 1 \mathrm{can}$ be considered as private, because they can only be accessed by the "private function" f2. However, we decided to not classify $\mathrm{f} 2$ as a private method, mainly because it cannot access the this object, nor can it be directly called from the public methods of the class.

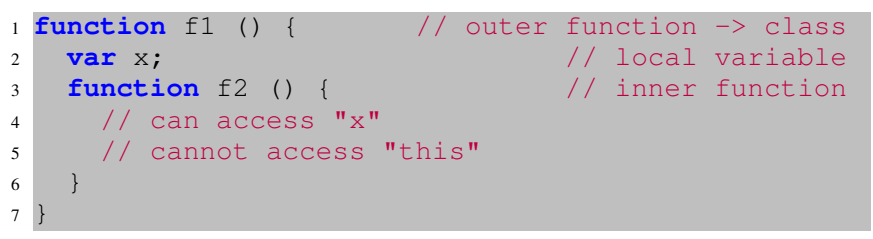

Listing 4. Using closures to implement "private" inner functions

In JavaScript, it is also possible to remove properties from objects dynamically, e.g., by calling delete myCircle.radius. Therefore, at runtime, an object can have less attributes than the ones defined in its class. It is also possible to modify the prototype chains dynamically. When we detect multiple assignments to the prototype link of a class A, a warning is raised reporting the alternative superclasses of A. Finally, the behavior of a program can be modified dynamically, using the eval operator [13], [14]. However, we do not consider the impact of eval's in the strategy described in Section III-A. For example, we do not account for classes entirely or partially created by means of eval.

Alexandre $>$ What happens with functions that partially match your class definition?

\section{STUDY}

The goal of this study is to evaluate whether the proposed strategy is able to detect classes in real Javascript software. The perspective is that of JavaScript developers interested in understanding and evolving the classes used in their systems. The context of this study consists of 50 popular Javascript systems, available at GitHub.

In this study, we answer the following research questions:

- RQ \#1: How often do JavaScript developers use classes?

- RQ \#2: How often do JavaScript developers use subclasses?

- RQ \#3: What is the size of JavaScript classes (in terms of number of methods and attributes)?

In the following, we first describe the process we followed to select JavaScript software from GitHub and to clean up the downloaded code (Section IV-A). Next, we present and discuss answers for the proposed research questions (Sections IV-B to IV-D). Finally, we discuss threats to validity (Section IV-E).

\section{A. Data Extraction}

The JavaScript systems considered in this study are available at GitHub. We selected systems ranked with at least 1,000 stars at GitHub, whose sole language is JavaScript, that have at least 150 commits and that are not forks of other projects. This search was performed on June, 2014 and resulted in 50 systems. After the check out of each system, we automatically inspected the source code to remove the following files: compacted files used in production to reduce network bandwidth consumption (which have the extension $*$.min.js), copyright files (copyright.js), documentation files (located in directories called doc or docs), and files belonging to third party libraries (located in directories thirdparty or node_modules). We did not discard test files and examples, because these files usually include new expressions, which are primordial for the success of the strategy proposed to detect classes, as described in Section III-A.

The selected systems are presented in Table II, including their version, a brief description, and size, in terms of lines of code and number of files. Although we did not discard test files and examples, they are not counted when computing the size metrics in Table II. The selection includes well-known and widely used JavaScript systems, from different domains, covering frameworks (e.g., angular.js and jasmine), editors (e.g., brackets), browser plug-ins (e.g., pdf.js), games (e.g., 2048 and clumsy-bird), etc. The largest system (ace) has 194,159 LOC and 574 files with .js extension. The smallest system (masonry) has 197 LOC and a single file. The average size and standard deviation is $13,846 \pm 33,720$ LOC and 58.68 \pm 121.1 files. The median is 2,462 LOC and 16 files. We also found systems with hundreds of functions in a single JavaScript file. For example, reveal.js is a system with a single file and 105 functions.

After downloading the systems and cleaning up the code, we executed the JSCLASSFINDER tool to extract class information and metrics on each system.

\section{B. How often JavaScript developers use classes?}

Table II presents the total number of classes detected by JSCLASSFINDER in the selected systems. We found classes in 37 out of 50 systems (74\%). The system with the largest number of classes is pdf.js (144 classes), followed by ace (133 classes), three.js (106 classes), and brackets (101 classes). Semantic-UI is the largest system (11,951 LOC) that does not have classes, at least as detect by our strategy. Systems in Table II are classified in ascending value of what we called Class Usage Ratio (CUR), which is defined as:

$$
\text { CUR }=\frac{\# \text { methods }+\# \text { classes }}{\# \text { functions }}
$$

This metric is the ratio of functions in a program that are related to the implementation of classes, i.e., that are methods or that are classes themselves. It ranges between 0 (system 
TABLE II

JAVASCRIPT SYSTEMS (ORDERED ON THE CUR COLUMN, SEE DESCRIPTION IN ACCOMPANYING TEXT)

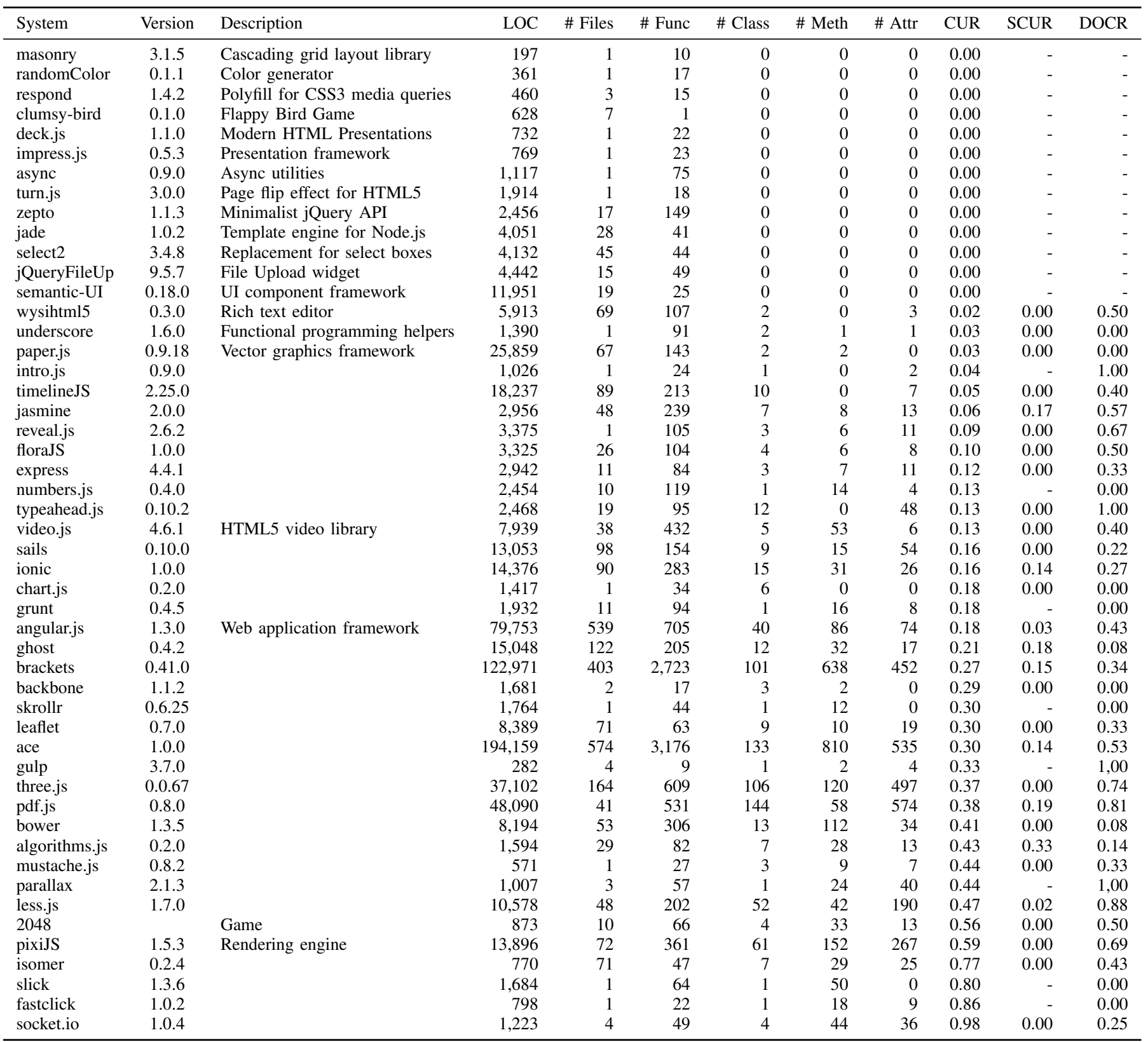

with no functions related to classes) to 1 (fully class-based system, where all functions are used to support classes). For systems that do not have functions, we define that $C U R$ is zero.

Figure 2 shows the distribution of the CUR values, considering all 50 systems (on the left and systems with CUR greater than zero on the right). On the left, there are systems with very small CUR values. The first quartile is 0.005 (lower bound of the black box within the "violin") and 13 systems have CUR equal to zero (the width of the "violin" is an indication of the number of the distribution of the systems for a given CUR value). The median for all systems (white dot at the heart of the violin) is 0.15 and the third quartile is 0.36 (upper bound of the black box). We also found one almost fully class-oriented system, socket.io, with CUR equal to 0.98 .

One sees a significant change in the CUR distribution when we only consider the systems with CUR greater than zero, as represented in the violin plot on the right. One could consider that these systems are those that chose to use classes with the convention that our tool is looking for. Other systems might use other conventions, or no class abstraction at all. The first quartile of CUR is now 0.13 , the median is 0.27 and the third quartile is 0.43 .

In other words, $26 \%$ of the systems do not use classes at all 
or are using another abstraction that the one we are looking for. This might be a deliberate design decision of their developers. On the other hand, in the remaining systems, the emulation of class is relevant, representing on the median $27 \%$ of the functions.

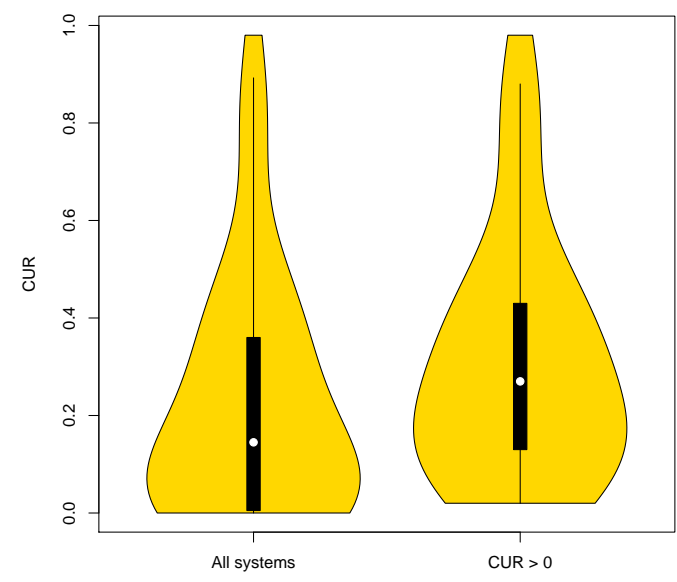

Fig. 2. Class Usage Ratio (CUR) distribution

We computed the Spearman's rank correlation coefficient between CUR and the following system size metrics: LOC, number of files, and number of functions. The intention is to clarify the effect of the system's size on the usage of classbased structures. The results are presented in Table III. We found a very weak correlation for LOC $(\rho=0.11$, p-value $=0.4)$, and number of files $(\rho=0.22$, $\mathrm{p}$-value $=0.1)$, and slightly better for number of functions $(\rho=0.29, \mathrm{p}$-value $=0.04)$. Marco $>L e o$, we will have to explain what the $p$-values mean ... The $p$-values are ....

TABLE III

SPEARMAN CORRELATION BETWEEN CUR AND SIZE METRICS

\begin{tabular}{llll}
\hline & LOC & \# Files & \# Func \\
\hline Spearman & 0.110 & 0.217 & 0.295 \\
p-value & 0.446 & 0.130 & 0.037 \\
\hline
\end{tabular}

In summary, we observed four main groups of systems:

- Class-free systems: 13 systems that do not use classes at all $(C U R=0)$.

- Class-agnostic systems: 18 systems that use classes, but marginally ( $C U R \leq 0.21$ ).

- Class-aware systems: 15 systems where classes represent an important and common data structure $(0.21<C U R<$ $0.59)$

- Class-oriented systems: four systems that can be classified as class-oriented systems ( $C U R \geq 0.77$ ).

Finally, the category of a system does not seem to be related to its size. Nic you could test that with a Kruskal Wallis ANOVA test: $\mathrm{HO}=$ median size of all categories are equal 4

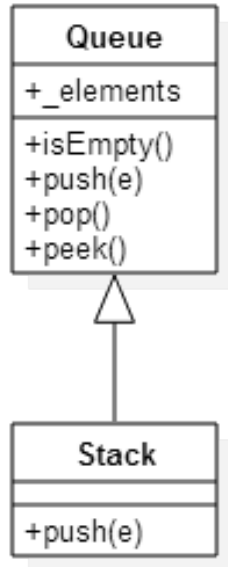

Fig. 3. Example of inheritance in algorithms.js

\section{How often JavaScript developers use subclasses?}

To evaluate the usage of inheritance, we propose a metric called Subclass Usage Ratio (SCUR), defined as:

$$
\text { SCUR }=\frac{\mid\{C \in \text { Classes } \mid \operatorname{DIT}(C) \geq 2\} \mid}{\mid \text { Classes } \mid-1}
$$

where Classes is the set of all classes in a given system. SCUR ranges from 0 (system that does not make use of inheritance) to 1 (system where all classes inherit from another class, except one class that is the root of the class hierarchy). SCUR is only defined for systems that have at least two classes.

As showed in Table II, the use of prototype-based inheritance is rare in JavaScript systems. First, we counted 29 systems (58\%) having two or more classes, i.e., systems where it is possible to detect the use of inheritance. However, in 20 of such systems $(69 \%)$, we did not found subclasses $(S C U R=0)$. The system with the highest use of inheritance is algorithms.js $(S C U R=0.33)$. As an example, in this system we found a class Stack defined as a subclass of Queue, as represented in the class diagram of Figure 3. In this example, Stack inherits three methods from Queue (isEmpty ()$, \operatorname{pop}()$, and peek ()$)$ and redefines one method $(\operatorname{push}())$.

D. What is the size of JavaScript classes (in terms of number of methods and attributes)?

To answer this question, we initially investigated only systems with at least 40 classes (7 systems), since they include a significant number of classes, compatible with medium-sized systems in class-based languages. Figure 4 shows the quantile functions for the Number of Attributes (NOA) and Number of Methods (NOM) values in each of these systems. In this figure, each black line represents a system. The $\mathrm{x}$-axis represents the quantiles and the $y$-axis represents the upper metric values for the classes in a given quantile. For example, suppose the value of a given quantile $p$ ( $\mathrm{x}$-axis) is $k$ (y-axis), for NOA values. This means that $\mathrm{p} \%$ of the classes in the system in question have at most $k$ attributes. Moreover, Figure 4 includes a red 


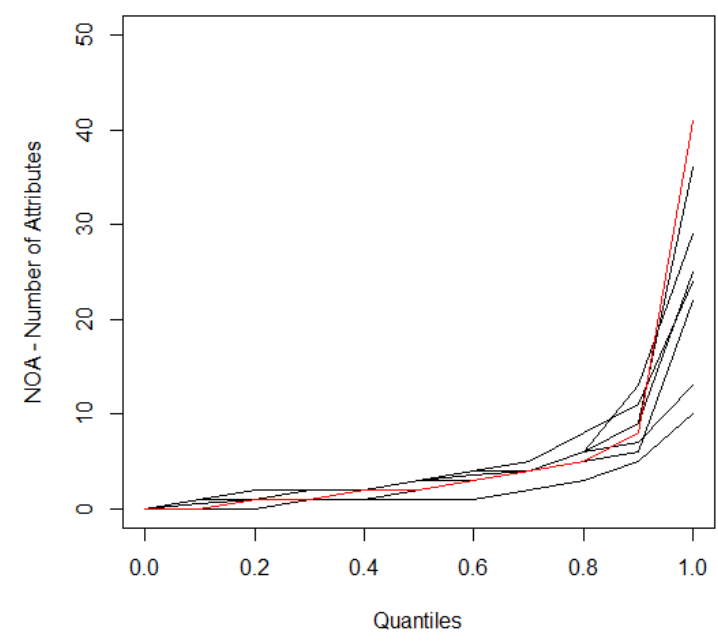

(a) NOA

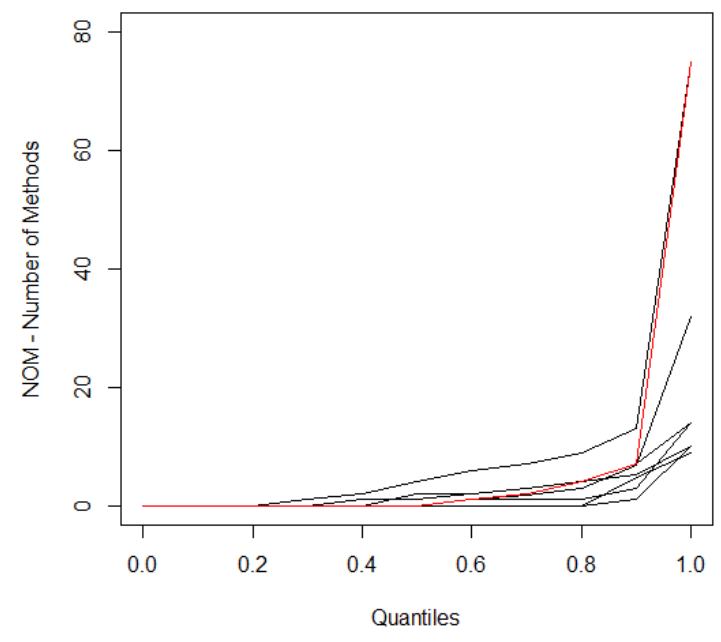

(b) NOM

Fig. 4. Quantile functions

line that represents the whole population of classes found in our dataset (787 classes, detected in 37 systems).

Regarding the NOA values, the quantile functions show that the vast majority of the classes have at most 11 attributes. Specifically, the 90th percentile values range from 5 attributes (angular.js) to 11 attributes (brackets and three.js). Regarding NOM values, the vast majority of the classes have less than 13 methods. In this case, the 90th percentile ranges from one method (pdf.js) to 13 methods (brackets). Regarding the whole population of classes, the results are similar. For NOA, the 90th percentile is $\mathrm{xx}$; for NOM, the 90th percentile is $\mathrm{xx}$.

In fact, when generating the quantile functions, we found a very large class in the ace system, with 164 attributes and 503 methods. By inspecting its source code, we discovered that this class is a PHP parser, automatically generated by a parser generator tool. For this reason, we removed this class from the quantile functions presented in Figure xx. Otherwise, it would require the presentation of very high values in the y-axis. Anyway, this finding shows that class-emulation in JavaScript is also a design decision followed by developers of program generators tools.

Figure 4 also shows that the considered metric values tend to present a right-skewed (or heavy-tailed) behavior, meaning that while the bulk of the distribution occurs for fairly small classes (in terms of NOA and NOM) there is a small number of large classes with NOA and NOM measures much higher than the typical value, producing a long tail to the right, if the metric values are presenting in a histogram. In fact, this heavy-tailed behavior is normally observed in source code metrics [15]-[17].

In Table II, 16 out of 37 systems with classes have a total number of attributes (\# Attr column) greater than the total number of methods (\# Meth column). This contrasts to the common shape of classes in class-based languages, when usually classes have more methods than attributes [18]. Therefore, we propose a metric called Data-Oriented Class Ratio (DOCR) to investigate this phenomena in more detail. $D O C R$ is defined as follows:

$$
\text { DOCR }=\frac{\mid\{C \in \text { Classes } \mid \operatorname{NOA}(C)>\operatorname{NOM}(C)\} \mid}{\mid \text { Classes } \mid}
$$

where Classes is the set of all classes in a given system. DOCR ranges from 0 (system where all classes have more attributes than methods) to 1 (system where the number of methods of each class is equal or greater than the number of attributes). $D O C R$ is only defined for systems that have at least one class.

Table II presents the DOCR values for each system and Figure 5 shows the DOCR distribution using a violin plot. The median DOCR value is $\mathrm{xx}$, which can be considered as a high measure. For example, metric thresholds for Java suggest that classes should have at most 8 attributes and 16 methods, i.e., they recommend two methods per attribute for a typical class [19]. On the other hand, half of the JavaScript systems we studied have more than $\mathrm{xx} \%$ of classes with more attributes than methods. Although we have not inspected the code in detail to explain this phenomena, we hypothesize that it might be due to the less importance that JavaScript gives to encapsulation. For this reason, getters and setters are usually more rare than in Java software.

\section{E. Threats to Validity}

First, the proposed strategy to detect classes do not handle the whole spectrum of class styles supported by JavaScript and 


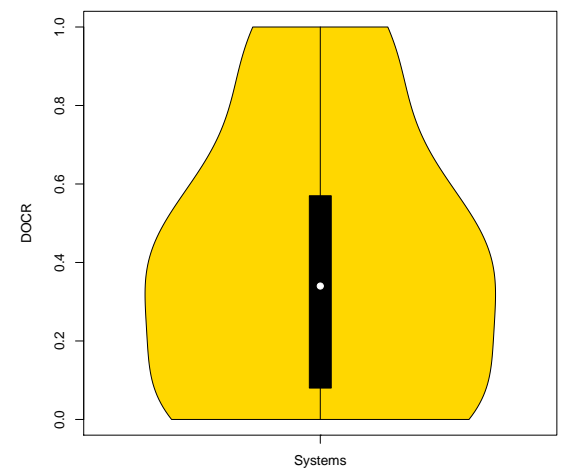

Fig. 5. Data-Oriented Classes Ratio (DOCR) distribution

also by third-party frameworks, as discussed in Section III-C. However, we cover the style that closely resemble the syntax and semantics of classes, attributes, and methods in class-based languages. Second, as usual, our dataset might not represent the whole population of JavaScript systems. But at least we selected a representative number of popular and well-known systems, of different sizes and covering various domains. Third, the proposed strategy depends on new expressions to detect classes. Therefore, it misses important classes of APIs, libraries, and frameworks, which are designed to be instantiated by clients. To mitigate this issue, we did not removed test files and files with examples from our dataset, when searching for classes. In a manual inspection, we did not found classes in such systems. However, if they exist, they are counted in our study.

\section{RELATED WORK}

Studies: Richards et al. [13] conducted a large-scale study on the use of eval in JavaScript, based on a corpus of more than 10,000 popular web sites. They report that eval is popular, but not necessarily evil. It is usually considered a best practice for example when loading scripts or data asynchronously. After this first study, restricted to eval's, the authors conducted a second study, when they investigated a broad range of JavaScript dynamic features [4]. They concluded for example that libraries often change the prototype links dynamically, but such changes are restricted to built-in types, like Object and Array, and changes in user-created types are more rare. The authors also report that most JavaScript programs do not delete attributes from classes dynamically. To some extend, these findings support the feasibility of using heuristics to extract class-like structures statically from JavaScript code, as proposed in this paper.

Tools: Gama et al. [8] identified five styles for implementing methods in JavaScript: inside/outside constructor functions using anonymous/non-anonymous functions and using prototypes. Their main goal was to implement an automated approach to normalizing JavaScript code to a single consistent objectoriented style. They claim that mixing styles in the same code may hinder program comprehension and make maintenance more difficult. The strategy proposed in this paper covers the fives styles proposed by the authors. Additionally, we also detect attributes and inheritance.

Fard and Mesbah [20] proposed a set of 13 JavaScript code smells, including generic smells (e.g., long functions and dead code) and smells specific to JavaScript (e.g., creating closures in loops and accessing this in closures). They also describe a tool, called JSNode, for detecting code smells based on a combination of static and dynamic analysis. Among the proposed patterns, only Request Banquet is directly related to class-emulation in JavaScript. In fact, this smell was originally proposed to class-based languages [21], [22], to refer to subclasses that do not use or override many elements from their superclasses. Interestingly, our strategy to detect classes opens the possibility to detect other well-known class-based code smells in JavaScript software, like Feature Envy, Large Class, Shotgun Surgery, Divergent Change, etc.

There is also a variety of tools and techniques for analyzing, improving, and understanding JavaScript code, including tools to prevent security attacks [23]-[25], to understand event-based interactions [26], [27], and to support refactorings [28], [29].

ECMAScript 6: ECMAScript is the standard definition of JavaScript [1]. ECMAScript 6 [7] is the next version of this standard, which is currently in frozen state and it is planned to be officially released in early $2015^{8}$. Interestingly, a syntactical support to classes is included in this new release. For example, it will support the following class definition:

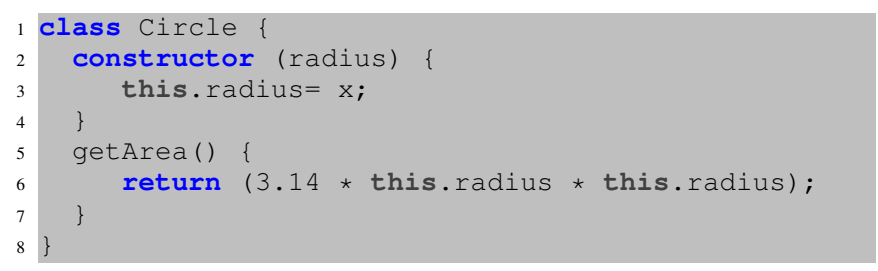

This support for classes was proposed to do not have an impact in the semantics of the language. For example, the previous class is equivalent to the following code:

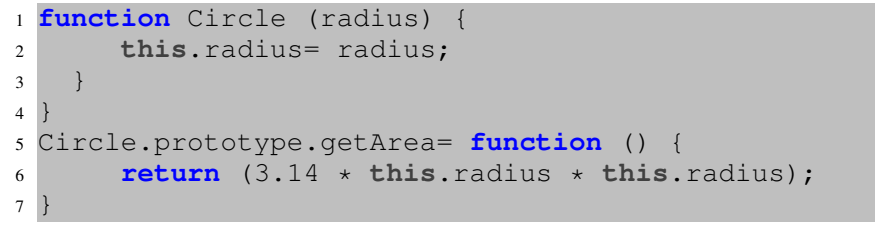

The strategy proposed in this paper straightforwardly detects this previous code as a Circle class, with a radius attribute and a getArea method, as specified in ECMAScript 6. Therefore, revealing how often JavaScript developers emulate classes in the current version of the language might be a valuable information for developers that plan to use classes in their new systems, according to the ECMAScript 6 standard. The proposed strategy and the JSCLASSFINDER tool can

\footnotetext{
${ }^{8}$ https://developer.mozilla.org/en-US/docs/Web/JavaScript/ New_in_JavaScript/ECMAScript_6_support_in_Mozilla, verified 11/15/2014
} 
also support a new variety of tools, aiming to translate "old JavaScript class styles" to ECMAScript 6 syntax.

\section{CONCLUSION}

Our tools and data are freely available at: http://aserg.labsoft.dcc.ufmg.br/jsclasses

\section{ACKNOWLEDGMENTS}

This research is supported by CNPq, CAPES, and FAPEMIG.

\section{REFERENCES}

[1] "European association for standardizing information and communi- cation systems (ECMA). ECMA-262: ECMAScript language specification. edition 5.1," 2011.

[2] H. Kienle, "It's about time to take JavaScript (more) seriously," IEEE Software, vol. 27, no. 3, pp. 60-62, May 2010.

[3] A. Nederlof, A. Mesbah, and A. van Deursen, "Software engineering for the web: the state of the practice," in 36th International Conference on Software Engineering (ICSE), Companion Proceedings, 2014, pp. 4-13.

[4] G. Richards, S. Lebresne, B. Burg, and J. Vitek, "An analysis of the dynamic behavior of JavaScript programs," in Conference on Programming Language Design and Implementation (PLDI), 2010, pp. $1-12$.

[5] A. H. Borning, "Classes versus prototypes in object-oriented languages," in ACM Fall Joint Computer Conference, pp. 36-40.

[6] A. Guha, C. Saftoiu, and S. Krishnamurthi, "The essence of JavaScript," in 24th European Conference on Object-Oriented Programming (ECOOP), 2010, pp. 126-150.

[7] "European association for standardizing information and communication systems (ECMA). ECMAScript language specification, 6th edition, draft october, 2014."

[8] W. Gama, M. Alalfi, J. Cordy, and T. Dean, "Normalizing object-oriented class styles in JavaScript," in 14th IEEE International Symposium on Web Systems Evolution (WSE), Sept 2012, pp. 79-83.

[9] D. Crockford, JavaScript: The Good Parts. O'Reilly, 2008.

[10] D. Flanagan, JavaScript: The Definitive Guide. O'Reilly, 2011.

[11] C. Anderson, P. Giannini, and S. Drossopoulou, "Towards type inference for JavaScript," in 19th European Conference on Object-Oriented Programming (ECOOP), 2005, pp. 428-452.

[12] S. R. Chidamber and C. F. Kemerer, "A metrics suite for object oriented design," IEEE Transactions on Software Engineering, vol. 20, no. 6, pp. 476-493, 1994.

[13] G. Richards, C. Hammer, B. Burg, and J. Vitek, "The eval that men do: A large-scale study of the use of eval in JavaScript applications," in 25th European Conference on Object-oriented Programming (ECOOP), 2011.

[14] F. Meawad, G. Richards, F. Morandat, and J. Vitek, "Eval begone!: Semiautomated removal of eval from Javascript programs," in Conference on Object Oriented Programming Systems Languages and Applications (OOPSLA), 2012, pp. 607-620.

[15] G. Baxter, M. Frean, J. Noble, M. Rickerby, H. Smith, M. Visser, H. Melton, and E. Tempero, "Understanding the Shape of Java Software," in International Conference on Object-oriented Programming Systems, Languages, and Applications (OOPSLA). ACM, 2006, pp. 397-412.

[16] P. Louridas, D. Spinellis, and V. Vlachos, "Power Laws in Software," ACM Transactions on Software Engineering and Methodology, vol. 18, pp. 1-26, 2008.

[17] R. Wheeldon and S. Counsell, "Power Law Distributions in Class Relationships," in International Working Conference on Source Code Analysis and Manipulation, 2003, pp. 45-54.

[18] R. Terra, L. F. Miranda, M. T. Valente, and R. S. Bigonha, "Qualitas.class Corpus: A compiled version of the Qualitas Corpus," Software Engineering Notes, vol. 38, no. 5, pp. 1-4, 2013.

[19] P. Oliveira, M. T. Valente, and F. Lima, "Extracting relative thresholds for source code metrics," in IEEE Conference on Software Maintenance, Reengineering and Reverse Engineering (CSMR-WCRE), 2014, pp. 254263.

[20] A. Fard and A. Mesbah, "JSNOSE: Detecting JavaScript code smells," in 13th Working Conference on Source Code Analysis and Manipulation (SCAM), 2013, pp. 116-125.
[21] M. Fowler, Refactoring: improving the design of existing code. AddisonWesley, 1999.

[22] M. Lanza and R. Marinescu, Object-oriented metrics in practice: using software metrics to characterize, evaluate, and improve the design of object-oriented systems. Springer, 2006.

[23] P. Vogt, F. Nentwich, N. Jovanovic, E. Kirda, C. Krügel, and G. Vigna, "Cross site scripting prevention with dynamic data tainting and static analysis," in Network and Distributed System Security Symposium (NDSS), 2007.

[24] A. Guha, S. Krishnamurthi, and T. Jim, "Using static analysis for Ajax intrusion detection," in 18th International Conference on World Wide Web $(W W W), 2009$, pp. 561-570.

[25] D. Yu, A. Chander, N. Islam, and I. Serikov, "JavaScript instrumentation for browser security," in 34th Symposium on Principles of Programming Languages (POPL), 2007, pp. 237-249.

[26] S. Alimadadi, S. Sequeira, A. Mesbah, and K. Pattabiraman, "Understanding JavaScript event-based interactions," in International Conference on Software Engineering (ICSE), 2014, pp. 367-377.

[27] A. Zaidman, N. Matthijssen, M. D. Storey, and rie van Deursen, "Understanding Ajax applications by connecting client and server-side execution traces," Empirical Software Engineering, vol. 18, no. 2, pp. 181-218, 2013

[28] A. Feldthaus, T. D. Millstein, A. Møller, M. Schäfer, and F. Tip, "Refactoring towards the good parts of JavaScript," in 26th Conference on Object-Oriented Programming (OOPSLA), Companion Proceedings, 2011, pp. 189-190.

[29] — "Tool-supported refactoring for JavaScript," in 26th Conference on Object-Oriented Programming, Systems, Languages, and Applications (OOPSLA), 2011, pp. 119-138. 